\title{
Neoadjuvant Chemoradiotherapy in Patients With Unresectable Locally Advanced Sigmoid Colon Cancer: Clinical Feasibility and Outcome
}

Shao-Qing Niu

Sun Yat-sen University Cancer Center

Rong-Zhen Li

Sun Yat-sen University Cancer Center

Yan Yuan

Sun Yat-sen University Cancer Center

Wei-Hao Xie

Sun Yat-sen University Cancer Center

Qiao-Xuan Wang

Sun Yat-sen University Cancer Center

Hui Chang

Sun Yat-sen University Cancer Center

Zhen-Hai Lu

Sun Yat-sen University Cancer Center

Pei-Rong Ding

Sun Yat-sen University Cancer Center

Li-Ren Li

Sun Yat-sen University Cancer Center

Xiao-Jun Wu

Sun Yat-sen University Cancer Center

\section{Zhi-Fan Zeng}

Sun Yat-sen University Cancer Center

Wei-Wei Xiao

Sun Yat-sen University Cancer Center

Yuan-Hong Gao ( $\sim$ gaoyh@sysucc.org.cn )

Sun Yat-sen University Cancer Center https://orcid.org/0000-0001-7459-2713

\section{Research}

Keywords: Sigmoid colon cancer, Neoadjuvant chemoradiotherapy, Down staging, Pathological complete response, Organ preservation 
Posted Date: February 13th, 2021

DOI: https://doi.org/10.21203/rs.3.rs-200191/v1

License: (c) (i) This work is licensed under a Creative Commons Attribution 4.0 International License. Read Full License 


\section{Abstract}

Background: Patients with locally advanced sigmoid colon cancer (LASCC) have limited treatment options and a dismal prognosis with poor quality of life. This prospective study aimed to further evaluate the feasibility and efficacy of neoadjuvant chemoradiotherapy (NACRT) followed by surgery as treatment for select patients with unresectable LASCC.

Methods: We studied patients with unresectable LASCC who received NACRT between November 2010 and April 2019. The NACRT regimen consisted of intensity modulated radiotherapy (IMRT) of 50 Gy to the gross tumor and positive lymphoma nodes and $45 \mathrm{~Gy}$ to the clinical target volume. Capecitabine-based chemotherapy was administered every 3 weeks. Surgery was scheduled 6-8 weeks after radiotherapy.

Results: Seventy-two patients were enrolled in this study. Patients had a regular follow-up (median, 41.1 months; range, 8.3-116.5 months). Seventy-one patients completed NACRT, and sixty-five completed surgery. Resection with microscopically negative margins (R0 resection) was achieved in 64 patients (88.9\%). Pathologic complete response was observed in 15 patients $(23.1 \%)$, and multivisceral resection was necessary in 38 patients (58.3\%). The cumulative probability of 3-year overall survival and disease-free survival were $75.8 \%$ and $70.7 \%$, respectively.

Conclusion: For patients with unresectable LASCC, neoadjuvant chemoradiotherapy is feasible, surgery can be performed safely and may result in increased survival and organ preservation rates.

\section{Background}

Colorectal cancer is the third most common malignancy and the third leading cause of cancer-related death worldwide [1]. Approximately $15 \%$ of patients present with locally advanced tumor (T4 stage), and if the tumor directly invades other organs or structures, multivisceral resection (MVR) is required [2]. Despite application of multiple treatment strategies, patients with locally advanced colon carcinoma (LACC) still have poor prognoses [3-7].

NACRT has been established as standard therapy for local advanced rectal cancer (LARC) [8] and may reduce local recurrence, but this has not been elucidated in colon cancer. Results of existing series studies of colon cancer show that neo-chemoradiotherapy can be beneficial for selected unresectable LACCs [914]. Our previous study[10] also proved that preoperative chemoradiotherapy and surgery can be performed safely and may result in an increased survival rate in patients with locally advanced sigmoid colon cancer (LASCC). In this study, we expand the sample size and prolong the follow-up time, and we described the treatment results of the adoption of neo-CRT for unresectable LASCC patients.

\section{Methods And Materials}

\section{Patient selection}


Patients with pathologically diagnosed and unresectable LASCC in our hospital between November 2010 and April 2019 were enrolled. This was an observational study approved by our institutional medical ethics committee (B2020-174-01). Patients with LASCC (defined as the primary tumor having an inferior margin $>15 \mathrm{~cm}$ from the anal verge) were selected to undergo neoadjuvant chemoradiotherapy (NACRT) on a case-by-case basis through multidisciplinary team consultation. This study had the following inclusion criteria: 1) curative resection was impossible due to preoperative imaging examinations showing that the tumor extensively involved adjacent organs/structures or involved multiple lymph node metastases, making radical resection difficult to achieve; 2 ) curative resection was deemed impossible after exploratory laparotomy. Patients with the following criteria were excluded: 1) patients with uncontrolled medical conditions (e.g., hypertension, diabetes, heart failure, or psychiatric disease); 2) prior history of other malignancies. This study was approved by the Institutional Review Board of Sun Yat-sen University Cancer Center. Before treatment, written informed consent was obtained from all patients.

\section{Treatment procedure details}

Radiotherapy (RT) was delivered using IMRT with $6 \mathrm{MV}$ photon beams, and all plans were calculated using the Eclipse or Monaco system. Median radiation doses were $50 \mathrm{~Gy}$ (range: 45.0-54.0 Gy) for gross tumor volume (GTV) and positive lymph nodes and $45 \mathrm{~Gy}$ (46.0-56.0 Gy) for clinical target volume (CTV) with conventional segmentation. Gross tumor volume (GTV) was defined as the macroscopic tumor and involved regional lymph nodes shown on imaging studies and physical examination before treatment. CTV as defined as GTV with a cranio-caudal margin of $2-3 \mathrm{~cm}$, sigmoid mesocolon and lymphatic drainage regions. If adjacent structures were involved, a further $1.5 \mathrm{~cm}$ isotropic margin into the involved structures and the ischiorectal fossa was included to account for microscopic disease and possible implantation metastases to the pelvic floor. Patients received capecitabine chemotherapy regimens during radiotherapy, and adjuvant chemotherapy consisted of a capecitabine-based regimen. Surgery was scheduled 6-8 weeks after RT. All imaging and blood tests were repeated before surgery. When tumor infiltration or adhesion to adjacent organs was detected intraoperatively, MVR was required.

\section{Response, toxicity and complications}

Acute and late adverse events were graded according to the Common Terminology Criteria for Adverse Events (version 4.03). Surgical complications were assessed according to the Clavien-Dindo classification [15].

\section{Follow-up}

Outpatient follow-up visits were performed every 3 months during the first 2 years after treatment, semiannually in the subsequent 3 years, and then yearly thereafter. Patients were followed up by outpatient interview or telephone until death or through May 31, 2020.

\section{Statistical analysis}


Statistical analysis was performed using Statistical Product and Service Solutions software for Windows (SPSS Inc., Version 25, Chicago, IL). Kaplan-Meier curves were used to calculate overall survival (OS), progression free survival (PFS), and local control (LC). PFS was defined as freedom of local, regional and distant failure from the date of diagnosis with biopsy confirmation to date of first documented relapse. Patients who were alive at last follow-up and progress free were censored. Primary end points were overall survival (OS) and progression-free survival (PFS). Secondary end points included tumor response grade (TRG) and the rate of RO.

\section{Results}

\section{Characteristics and compliance}

Between November 2010 and April 2019, a total of 72 patients were enrolled in this study. The mean age was 56 (range: 29-79) years old, and 75\% ( $n=54)$ were males. According to the 8th edition of the Union for American Joint Cancer Committee (AJCC) TNM staging system, 9 patients were diagnosed with T3 stage, 5 with T4a and 58 with T4b disease. Three quarters of pathologic diagnoses were moderately differentiated adenocarcinoma. The most commonly involved organs were bladder (62.5\%), abdominal/pelvic wall (25.0\%), small intestine (12.5\%) and ureter (11.1\%). Among the 45 patients with bladder involvement, six were proved by cystoscopy and the rest by CT and/or MRI and irritation signs of the bladder (e.g. hematuria, urgent urination, frequent micturition, odynuria). Clinical and treatment characteristics of study patients are presented in Table 1.

\section{Short-term clinical efficacy}

After NACRT, 65 patients with initially unresectable tumors were successfully transformed to operable, while tumors in 7 patients failed to reach this criterion. Among the 65 patients undergoing surgery, 64 $(64 / 72,88.89 \%)$ patients who received radical surgical resection with negative margins (R0), and one exhibited macroscopic residue (R2). Fifteen patents (23.1\%) experienced pathological complete remission (pCR) after NACRT. Among the 45 patients with bladder invasion before treatment, only two received complete bladder excision, 21 received partial bladder excision, and 15 patients' bladders were completely preserved (the remains 7 patients abandoned surgery). Surgical results and pathological findings are detailed in Table 2.

Among the 65 patients who received surgery, 64 had R0 resection (64/72, 88.9\%), and 15 (20.8\%) achieved pathological complete remission after NACRT.

\section{Long-term survival}

Median follow-up of surviving patients was 41.1 months (range, 8.3-116.5 months) in the entire group. The estimated 3-year OS, PFS, RFS and MFS were 75.8\%, 70.7\%, 89.0\%, and 75.2\%, respectively (Figure 2). 
In univariate analysis, non-R0 resection, non-downstaging T, postsurgical pathology N stage (N1), postsurgical pathology $\mathrm{N}$ stage (T4a-T4b), low differentiation and perineurium invasion (PNI) were significantly associated with poorer $\mathrm{OS}$, while non-RO resection, no pathological complete remission (no$\mathrm{PCR}$ ), non-downstaging T, postsurgical pathology $\mathrm{N}$ stage (T4a-T4b) and PNI were associated with reduced PFS $(p<0.05)$ (Table 3$)$. In multivariate analysis, differentiation remained an independent prognostic factor for overall survival rates (Figure 3A). Meanwhile, downstaging $T$ was an independent prognostic factor for PFS (Figure 3B).

\section{Treatment-related toxicity}

Treatment toxicities were assessed according to CTCAE criteria version 4.03 as shown in Table 4 . The most common NACRT-related toxicities were grade 1 to 2 myelosuppression (88.9\%), mucositis/dermatitis (97.2\%) and gastrointestinal (GI) toxicities (93.1\%). Four patients developed intestinal obstruction during NACRT. Only one patient failed to complete the radiation course due to tumor rupture and underwent emergency surgery. Among the 65 patients who underwent surgery, grade 3/4 Clavien-Dindo postsurgical complications were observed in 5 cases (7.7\%).

\section{Discussion}

Although NACRT is the standard treatment for local advanced rectal cancer, its place in the management of sigmoid colon cancer has yet to be defined. There are several single or very small sample size case reports about utilizing NACRT for sigmoid colon cancer [12, 16-18]. M Cukier et al [18] retrospectively reviewed 33 patients with potentially resectable, non-metastatic primary LACC who received neoadjuvant CRT, and all patients had RO resection. The rates of pCR and 3-year OS were $3 \%$ and $85.9 \%$, respectively. Our previous work [10] also revealed promising clinical outcomes and mild side effects in response to NACRT in LASCC, in which all 21 LASCC patients (100\%) with locally unresectable disease attained resectable disease, including 14 patients $(66.7 \%)$ who received a simple colectomy and 7 patients (22.2\%) who were in need of MVR. The rates of PCR and 3-year OS were $38.1 \%$ and $95.2 \%$, respectively.

MVR is the recommended surgical treatment for LACC [19]. Mohan et al [20] found MVR to be associated with the best chance of long term survival when clear margins are achieved, and R0 resection was the strongest factor associated with long-term survival when analyzing 22 studies comprising 1575 patients from 1995 to 2012. Therefore, whether LACC patients could be successfully transformed from unresectable to resectable status is crucial for the goal of cure. NACRT provides patients with unresectable LASCC a choice to improve resectability and survival. Ideal treatment results were also seen in our study in 65 patients (90.3\%) who successfully transformed to resectable status, with R0 resection achieved in 64 patients (88.9\%). After a median follow-up of 41.1 months, patients presented with an OS of $75.8 \%$.

In our study, NACRT achieved satisfactory clinical outcomes. Sixty-five patients received surgical resection after NACRT. According to postoperative pathological results, 62 patients experienced downstaging, and the $\mathrm{PCR}$ rate was as high as $23.1 \%$. T stage was down-graded in 54 patients $(75.0 \%)$, and the 
$\mathrm{N}$ stage was down-graded in 63 cases (87.5\%). In fact, when followed up for a median period of 41.1 months, the 3-year OS and PFS were $75.8 \%$ and $70.7 \%$, respectively, which is comparable to results recorded in the literature $[9,10,21]$.

In addition to improving the prognosis, NACRT ameliorates organ preservation during surgery. The bladder and small intestine are the most commonly affected organs in LASCC, which are most commonly removed in MVR. In this study, 45 patients (62.5\%) exhibited bladder invasion before treatment. Among the 65 patients receiving surgery, only 21 cases $(32.3 \%)$ received partial cystectomy, while two received total cystectomy. Owing to NACRT, 36 patients $(36 / 45,80 \%)$ retained bladder function. Therefore, NACRT improved quality of life in these patients by preserving important organs.

Acute toxicities in response to NACRT were mild. Myelosuppression and radiodermatitis/mucositis were the most common adverse events. For myelosuppression, grade 1-2 incidence was $88.9 \%$, and grade $3-4$ was $11.1 \%$. The incidences of grade $1-2$ and grade $3-4$ mucositis and dermatitis were $97.2 \%$ and $2.8 \%$, respectively. Only two patients experienced grade 3-4 gastrointestinal reactions. Therefore, according to the results of this study, NACRT for LASCC is both safe and tolerable.

There are several limitations to this study. First, the sample size was small, and the median follow-up period of 41.1 months was rather short. Second, this study was non-randomized.

\section{Conclusions}

NACRT is feasible in patients with unresectable LASCC, and surgery can be performed safely and may result in increased survival and organ preservation rates.

\section{Declarations}

- Ethics approval and consent to participate区This was an observational study was performed in accordance with the Declaration of Helsinki and approved by Sun Yat-sen University cancer center medical ethics committee (B2020-174-01). This study was approved by the Institutional Review Board of Sun Yat-sen University Cancer Center. Before treatment, written informed consent was obtained from all patients.

- Consent for publication: Not applicable, because our manuscript does not contain data from any individual person.

- Availability of data and material: The datasets analyzed during the current study available from the corresponding author on reasonable request.

- Competing interests $\bigotimes T$ The authors report no competing interests in this work.

- Funding $₫$ This work was supported by the Grants from National Natural Science Foundation of China ه81672987ه82073329『and Natural Science Foundation of Guangdong Province \2020A1515011286》. 
- Authors' contributions $₫$ Shao-Qing Niu, Rong-Zhen Li and Yan Yuan drafted the manuscript; Wei-Hao Xie, Qiao-Xuan Wang and Hui Chang performed the statistic analysis; Zhen-Hai Lu, Pei-Rong Ding, LiRen Li and Xiao-Jun Wu were members of the research group and participated in the treatment of patients and data collection; Zhi-Fan Zeng revised the draft; Wei-Wei Xiao and Yuan-Hong Gao conceived the study and participated in its design and coordination. All authors read and approved the final manuscript.

- Acknowledgements $\llbracket$ Not applicable.

\section{References}

1. Siegel RL, Miller KD, Jemal A: Cancer statistics, 2020. CA Cancer J Clin 2020, 70(1):7-30.

2. Klaver CEL, Kappen TM, Borstlap WAA, Bemelman WA, Tanis PJ: Laparoscopic surgery for T4 colon cancer: a systematic review and meta-analysis. Surg Endosc 2017, 31(12):4902-4912.

3. Li L, Li W, Wang C, Yan X, Wang Y, Niu C, Zhang X, Li M, Tian H, Yao C et al: Adoptive transfer of natural killer cells in combination with chemotherapy improves outcomes of patients with locally advanced colon carcinoma. Cytotherapy 2018, 20(1):134-148.

4. Klaver CEL, Wisselink DD, Punt CJA, Snaebjornsson P, Crezee J, Aalbers AGJ, Brandt A, Bremers AJA, Burger JWA, Fabry HFJ et al: Adjuvant hyperthermic intraperitoneal chemotherapy in patients with locally advanced colon cancer (COLOPEC): a multicentre, open-label, randomised trial. Lancet Gastroenterol Hepatol 2019, 4(10):761-770.

5. Jakobsen A, Andersen F, Fischer A, Jensen LH, Jorgensen JC, Larsen O, Lindebjerg J, Ploen J, Rafaelsen SR, Vilandt J: Neoadjuvant chemotherapy in locally advanced colon cancer. A phase II trial. Acta Oncol 2015, 54(10):1747-1753.

6. Van Cutsem E, Yoshino T, Lenz HJ, Lonardi S, Falcone A, Limon ML, Saunders M, Sobrero A, Park YS, Ferreiro $\mathrm{R}$ et al: Nintedanib for the treatment of patients with refractory metastatic colorectal cancer (LUME-Colon 1): a phase III, international, randomized, placebo-controlled study. Ann Oncol 2018, 29(9):1955-1963.

7. Kitano S, Inomata M, Mizusawa J, Katayama H, Watanabe M, Yamamoto S, Ito M, Saito S, Fujii S, Konishi $\mathrm{F}$ et al: Survival outcomes following laparoscopic versus open D3 dissection for stage II or III colon cancer (JCOG0404): a phase 3, randomised controlled trial. Lancet Gastroenterol Hepatol 2017, 2(4):261-268.

8. Sauer R, Liersch T, Merkel S, Fietkau R, Hohenberger W, Hess C, Becker H, Raab HR, Villanueva MT, Witzigmann $\mathrm{H}$ et al: Preoperative versus postoperative chemoradiotherapy for locally advanced rectal cancer: results of the German CAO/ARO/AIO-94 randomized phase III trial after a median follow-up of 11 years. J Clin Oncol 2012, 30(16):1926-1933.

9. Chang H, Yu X, Xiao WW, Wang QX, Zhou WH, Zeng ZF, Ding PR, Li LR, Gao YH: Neoadjuvant chemoradiotherapy followed by surgery in patients with unresectable locally advanced colon cancer: a prospective observational study. Onco Targets Ther 2018, 11:409-418. 
10. Qiu B, Ding PR, Cai L, Xiao WW, Zeng ZF, Chen G, Lu ZH, Li LR, Wu XJ, Mirimanoff RO et al: Outcomes of preoperative chemoradiotherapy followed by surgery in patients with unresectable locally advanced sigmoid colon cancer. Chin J Cancer 2016, 35(1):65.

11. Martenson JA, Jr., Willett CG, Sargent DJ, Mailliard JA, Donohue JH, Gunderson LL, Thomas CR, Jr., Fisher B, Benson AB, 3rd, Myerson R et al: Phase III study of adjuvant chemotherapy and radiation therapy compared with chemotherapy alone in the surgical adjuvant treatment of colon cancer: results of intergroup protocol 0130. J Clin Oncol 2004, 22(16):3277-3283.

12. Yoshitomi M, Hashida H, Nomura A, Ueda S, Terajima H, Osaki N: [A case of locally advanced sigmoid colon cancer treated with neoadjuvant chemoradiotherapy]. Gan To Kagaku Ryoho 2014, 41(9):1175-1178.

13. O'Neill B, Brown G, Wotherspoon A, Burton S, Norman A, Tait D: Successful downstaging of high rectal and recto-sigmoid cancer by neo-adjuvant chemo-radiotherapy. Clin Med Oncol 2008, 2:135144.

14. Hallet J, Zih FS, Lemke M, Milot L, Smith AJ, Wong CS: Neo-adjuvant chemoradiotherapy and multivisceral resection to optimize RO resection of locally recurrent adherent colon cancer. Eur $J$ Surg Oncol 2014, 40(6):706-712.

15. Dindo D, Demartines N, Clavien PA: Classification of surgical complications: a new proposal with evaluation in a cohort of 6336 patients and results of a survey. Ann Surg 2004, 240(2):205-213.

16. Fujie Y, Adachi K, Nonaka R, Moon JH, Hashimoto K, Fujita S, Ohnishi T: [A Case of Advanced Sigmoid Colon Cancer That Was Resected after Chemoradiation Therapy Following Ineffective Chemotherapy]. Gan To Kagaku Ryoho 2018, 45(13):2027-2029.

17. Burton S, Brown G, Daniels I, Norman A, Swift I, Abulafi M, Wotherspoon A, Tait D: MRI identified prognostic features of tumors in distal sigmoid, rectosigmoid, and upper rectum: treatment with radiotherapy and chemotherapy. Int J Radiat Oncol Biol Phys 2006, 65(2):445-451.

18. Cukier M, Smith AJ, Milot L, Chu W, Chung H, Fenech D, Herschorn S, Ko Y, Rowsell C, Soliman H et al: Neoadjuvant chemoradiotherapy and multivisceral resection for primary locally advanced adherent colon cancer: a single institution experience. Eur J Surg Oncol 2012, 38(8):677-682.

19. Chang GJ, Kaiser AM, Mills S, Rafferty JF, Buie WD, Standards Practice Task Force of the American Society of C, Rectal S: Practice parameters for the management of colon cancer. Dis Colon Rectum 2012, 55(8):831-843.

20. Mohan HM, Evans MD, Larkin JO, Beynon J, Winter DC: Multivisceral resection in colorectal cancer: a systematic review. Ann Surg Oncol 2013, 20(9):2929-2936.

21. Rashtak S, Ruan X, Druliner BR, Liu H, Therneau T, Mouchli M, Boardman LA: Peripheral Neutrophil to Lymphocyte Ratio Improves Prognostication in Colon Cancer. Clin Colorectal Cancer 2017, 16(2):115123 e113.

\section{Tables}


Table 1. Baseline clinicopathologic characteristics of the 72 patients with unresectable sigmoid colon cancer (LASCC). 


\begin{tabular}{|c|c|}
\hline Characteristic & No. (\%) \\
\hline \multicolumn{2}{|l|}{ Age } \\
\hline$\leq 65$ & $58 \rrbracket 80.6 \rrbracket$ \\
\hline$>65$ & $14 \rrbracket 19.4 \rrbracket$ \\
\hline \multicolumn{2}{|l|}{ Gender } \\
\hline Male & $54 \bigotimes 75.0 \bigotimes$ \\
\hline Female & $18 \rrbracket 25.0 \rrbracket$ \\
\hline \multicolumn{2}{|l|}{ cT stage } \\
\hline T3 & $9 \rrbracket 12.5 \rrbracket$ \\
\hline $\mathrm{T} 4 \mathrm{a}$ & $5 \rrbracket 6.9 \rrbracket$ \\
\hline $\mathrm{T} 4 \mathrm{~b}$ & $58 \rrbracket 80.6 \rrbracket$ \\
\hline \multicolumn{2}{|l|}{ cN stage } \\
\hline No & 1ब1.4区 \\
\hline N1 & $25 \rrbracket 34.7 \rrbracket$ \\
\hline N2 & $46 \rrbracket 63.9 \rrbracket$ \\
\hline \multicolumn{2}{|l|}{ Clinical stage } \\
\hline IIc & $1 \otimes 1.4 \rrbracket$ \\
\hline Illb & $15 \rrbracket 20.8 \rrbracket$ \\
\hline IIIc & $55 \rrbracket 76.4 \bigotimes$ \\
\hline IV & $1 \otimes 1.4 \rrbracket$ \\
\hline \multicolumn{2}{|l|}{ Tumor differentiation } \\
\hline High & $15 \rrbracket 20.8 \rrbracket$ \\
\hline Moderate & $54 \rrbracket 75.0 \bigotimes$ \\
\hline Low & $3 \llbracket 4.2 \rrbracket$ \\
\hline \multicolumn{2}{|l|}{ Involved organ } \\
\hline Bladder & $45 \llbracket 62.5 \rrbracket$ \\
\hline Ureter & $8 \otimes 11.1 \rrbracket$ \\
\hline Abdominal/Pelvic wall & $18 \rrbracket 25.0 \otimes$ \\
\hline Small intestine & $9 \rrbracket 12.5 \rrbracket$ \\
\hline
\end{tabular}

Page 11/19 


\begin{tabular}{|c|c|}
\hline \multicolumn{2}{|l|}{ CEA } \\
\hline$\leq 5 \mathrm{ng} / \mathrm{ml}$ & $30 \otimes 41.7 \rrbracket$ \\
\hline$>5 \mathrm{ng} / \mathrm{ml}$ & $35 \rrbracket 48.6 \rrbracket$ \\
\hline unknown & $7 \otimes 9.7 \rrbracket$ \\
\hline \multicolumn{2}{|c|}{ Bladder fistula/perforation } \\
\hline Yes & $14 \rrbracket 19.4 \rrbracket$ \\
\hline No & $58 \rrbracket 80.6 \rrbracket$ \\
\hline \multicolumn{2}{|c|}{ Intestinal obstruction } \\
\hline Yes & $13 \otimes 18.1 \rrbracket$ \\
\hline No & $59 ه 81.9 \rrbracket$ \\
\hline \multicolumn{2}{|c|}{ Family history } \\
\hline Yes & 12囚16.7》 \\
\hline No & $60 \rrbracket 83.3 \rrbracket$ \\
\hline \multicolumn{2}{|l|}{ MMR } \\
\hline dMMR & $6 \rrbracket 8.3 \rrbracket$ \\
\hline pMMR & $40 \rrbracket 55.6 \rrbracket$ \\
\hline Unknown & $26 \rrbracket 36.1 \rrbracket$ \\
\hline \multicolumn{2}{|l|}{ KPS } \\
\hline$\geq 90$ & $58 \rrbracket 80.6 \rrbracket$ \\
\hline$<90$ & 14囚19.4凶 \\
\hline
\end{tabular}

Abbreviations: KPS, Karnofsky Performance Status; BMI, Body Mass Index; cT stage, clinical T stage; cN stage, clinical $\mathrm{N}$ stage; MMR, mismatch repair phenotype.

Table 2. Treatment outcomes of surgeries and pathological findings in 72 surgical patients with LASCC. 


\begin{tabular}{|c|c|c|}
\hline & Outcomes & No. (\%) \\
\hline \multicolumn{3}{|c|}{ Surgery situation } \\
\hline & Ro & $64 \rrbracket 88.9 \rrbracket$ \\
\hline & R2 & $1 \otimes 1.4 \rrbracket$ \\
\hline & Abandoned & $7 \otimes 9.7 \rrbracket$ \\
\hline \multicolumn{3}{|c|}{ pT stage } \\
\hline & TO & 10ه13.9ه \\
\hline & T1 & $2 \varangle 2.8 \rrbracket$ \\
\hline & $\mathrm{T} 2$ & $7 \rrbracket 9.7 \rrbracket$ \\
\hline & T3 & $26 \rrbracket 36.1 \rrbracket$ \\
\hline & T4a & $6 \rrbracket 8.3 \rrbracket$ \\
\hline & $\mathrm{T} 4 \mathrm{~b}$ & $14 \rrbracket 19.4 \rrbracket$ \\
\hline \multicolumn{3}{|c|}{ pN stage } \\
\hline & No & $62 \rrbracket 86.1 \rrbracket$ \\
\hline & $\mathrm{N} 1$ & $3 \llbracket 4.2 \rrbracket$ \\
\hline & N2 & 0 \\
\hline \multicolumn{3}{|c|}{ Downstage T } \\
\hline & yes & $54 \bigotimes 75.0 \bigotimes$ \\
\hline & no & $11 \otimes 15.3 \rrbracket$ \\
\hline \multicolumn{3}{|c|}{ Downstage N } \\
\hline & yes & $63 \rrbracket 87.5 \rrbracket$ \\
\hline & no & $2 \varangle 2.8 \rrbracket$ \\
\hline \multicolumn{3}{|c|}{ Downstage } \\
\hline & yes & $62 \varangle 95.4 \rrbracket$ \\
\hline & no & $3 \rrbracket 4.6 \rrbracket$ \\
\hline \multicolumn{3}{|l|}{ MVR } \\
\hline & yes & $38 \otimes 58.5 \rrbracket$ \\
\hline & No & $27 \rrbracket 41.5 \rrbracket$ \\
\hline pCR & & \\
\hline
\end{tabular}

Page 13/19 


\begin{tabular}{|c|c|c|}
\hline & yes & $15 \bigotimes 23.1 \rrbracket$ \\
\hline & No & 50ه76.9ه \\
\hline \multicolumn{3}{|l|}{ TRG } \\
\hline & 1 & $11 \rrbracket 25.6 \rrbracket$ \\
\hline & 2 & $12 \rrbracket 27.9 \rrbracket$ \\
\hline & 3 & $13 \bowtie 30.2 \rrbracket$ \\
\hline & 4 & $7 \otimes 16.3 \rrbracket$ \\
\hline
\end{tabular}

Abbreviations: $\mathrm{pT}$ stage, postoperative pathology T stage; $\mathrm{pN}$ stage, postoperative pathology $\mathrm{N}$ stage; MVR, multivisceral resection; pCR, Pathologic complete remission; NA: not available; TRG: tumor regression grading.

Notes: \$ exploratory laparotomy;

* Four patients who underwent exploratory laparotomy were included in this group.

Table 3. Univariate and multivariable Cox analysis of prognostic factors for overall survival and progression free survival in 72 patients with unresectable sigmoid colon cancer (LASCC) treated with neoadjuvant chemoradiotherapy and surgery. 


\begin{tabular}{|c|c|c|c|c|}
\hline & $p$ & $\mathrm{HR}(95 \% \mathrm{Cl})$ & $p$ & $\mathrm{HR}(95 \% \mathrm{Cl})$ \\
\hline $\begin{array}{l}\text { overall survival } \\
\text { variable }\end{array}$ & & & & \\
\hline $\begin{array}{l}\text { R0 resection } \\
\text { 『R0 vs No-R0『 }\end{array}$ & $<0.001$ & $0.292 \rrbracket 0.184-0.465 \rrbracket$ & 0.973 & 0.937ه0.020-42.855囚 \\
\hline $\begin{array}{l}\text { pCR } \\
\text { 『pCR vs No pCR }\end{array}$ & 0.172 & $2.342 \rrbracket 0.690-7.946 \rrbracket$ & 0.339 & $0.194 \llbracket 0.007-5.597 \rrbracket$ \\
\hline $\begin{array}{l}\text { Down T stage } \\
\text { 『Yes vs No囚 }\end{array}$ & 0.044 & 3.031ه1.033-8.894】 & 0.426 & 2.021ه0.358-11.419囚 \\
\hline $\begin{array}{l}\text { pN Stage group } \\
\text { (pN0 vs pN1) }\end{array}$ & 0.037 & $5.234 ه 1.105-24.800 \rrbracket$ & 0.601 & $2.845 \rrbracket 0.057-142.977 \rrbracket$ \\
\hline $\begin{array}{l}\text { рT Stage group } \\
\text { (рT0-T3 vs pT4a-4b) }\end{array}$ & 0.023 & $2.678 \rrbracket 1.144-6.272 \rrbracket$ & 0.634 & $0.659 \llbracket 0.118-3.664 \rrbracket$ \\
\hline Differentiation & 0.001 & $0.062 \varangle 0.012-0.319 \rrbracket$ & 0.003 & $36.443 \rrbracket 3.500-379.429 \rrbracket$ \\
\hline PNI $\otimes$ Yes vs No囚 & 0.011 & 4.138ه1.377-12.435区 & 0.875 & 1.205ه0.119-12.166】 \\
\hline & $p$ & $\mathrm{HR}(95 \% \mathrm{Cl})$ & $p$ & $\mathrm{HR}(95 \% \mathrm{Cl})$ \\
\hline $\begin{array}{l}\text { progress free } \\
\text { survival variable }\end{array}$ & & & & \\
\hline $\begin{array}{l}\text { R0 resection } \\
\text { 『R0 vs No-R0囚 }\end{array}$ & $<0.001$ & $0.343 \rrbracket 0.219-0.538 \rrbracket$ & 0.232 & $0.091 \rrbracket 0.002-4.629 \rrbracket$ \\
\hline $\begin{array}{l}\text { pCR } \\
\text { 『Yes vs No囚 }\end{array}$ & 0.035 & 3.697ه0.865-15.806ه & 0.991 & 497046.093ه0.000- $\rrbracket$ \\
\hline Down T stage & 0.006 & 4.109ه1.490-11.333囚 & 0.027 & 6.095ه1.228-30.253囚 \\
\hline $\begin{array}{l}\text { рT Stage group } \\
\text { (рT0-T3 vs pT4a-4b) }\end{array}$ & 0.0101 & 2.988囚1.293-6.907》 & 0.797 & $0.818 \rrbracket 0.177-3.786 \rrbracket$ \\
\hline Differentiation & 0.111 & 2.292邓0.827-6.356ه & 0.130 & $4.246 \rrbracket 0.653-27.599 \rrbracket$ \\
\hline PNIIYYes vs No囚 & 0.015 & $3.883 \rrbracket 1.300-11.597 \rrbracket$ & 0.251 & $3.108 \otimes 0.448-21.581 \rrbracket$ \\
\hline
\end{tabular}


Table 4. NACRT toxicities and surgical complications in the 72 patients with unresectable sigmoid colon cancer (LASCC).

\begin{tabular}{|c|c|}
\hline Adverse effects & No. (\%) \\
\hline \multicolumn{2}{|l|}{ Myelosuppression } \\
\hline grade $0-2$ & $64 \rrbracket 88.9 \rrbracket$ \\
\hline grade $3-4$ & 8\11.1】 \\
\hline \multicolumn{2}{|l|}{ Mucositis/dermatitis } \\
\hline grade $0-2$ & $70 \otimes 97.2 \otimes$ \\
\hline grade $3-4$ & $2 \rrbracket 2.8 \rrbracket$ \\
\hline \multicolumn{2}{|l|}{ Gl toxicities } \\
\hline grade $0-2$ & $67 \rrbracket 93.1 \rrbracket$ \\
\hline grade $3-4$ & $5 \rrbracket 6.9 \rrbracket$ \\
\hline \multicolumn{2}{|l|}{ Intestinal obstruction } \\
\hline yes & $4 \rrbracket 5.6 \rrbracket$ \\
\hline no & $68 \varangle 94.4 \rrbracket$ \\
\hline \multicolumn{2}{|l|}{ Anastomotic leakage } \\
\hline yes & $9 \rrbracket 12.5 \rrbracket$ \\
\hline no & $63 \llbracket 87.5 \rrbracket$ \\
\hline Nonresectable surgery \# & 7 \\
\hline
\end{tabular}

Abbreviations: $\mathrm{Gl}$, gastrointestinal.

Notes: \# Including 7 cases who abandoned surgery.

\section{Figures}




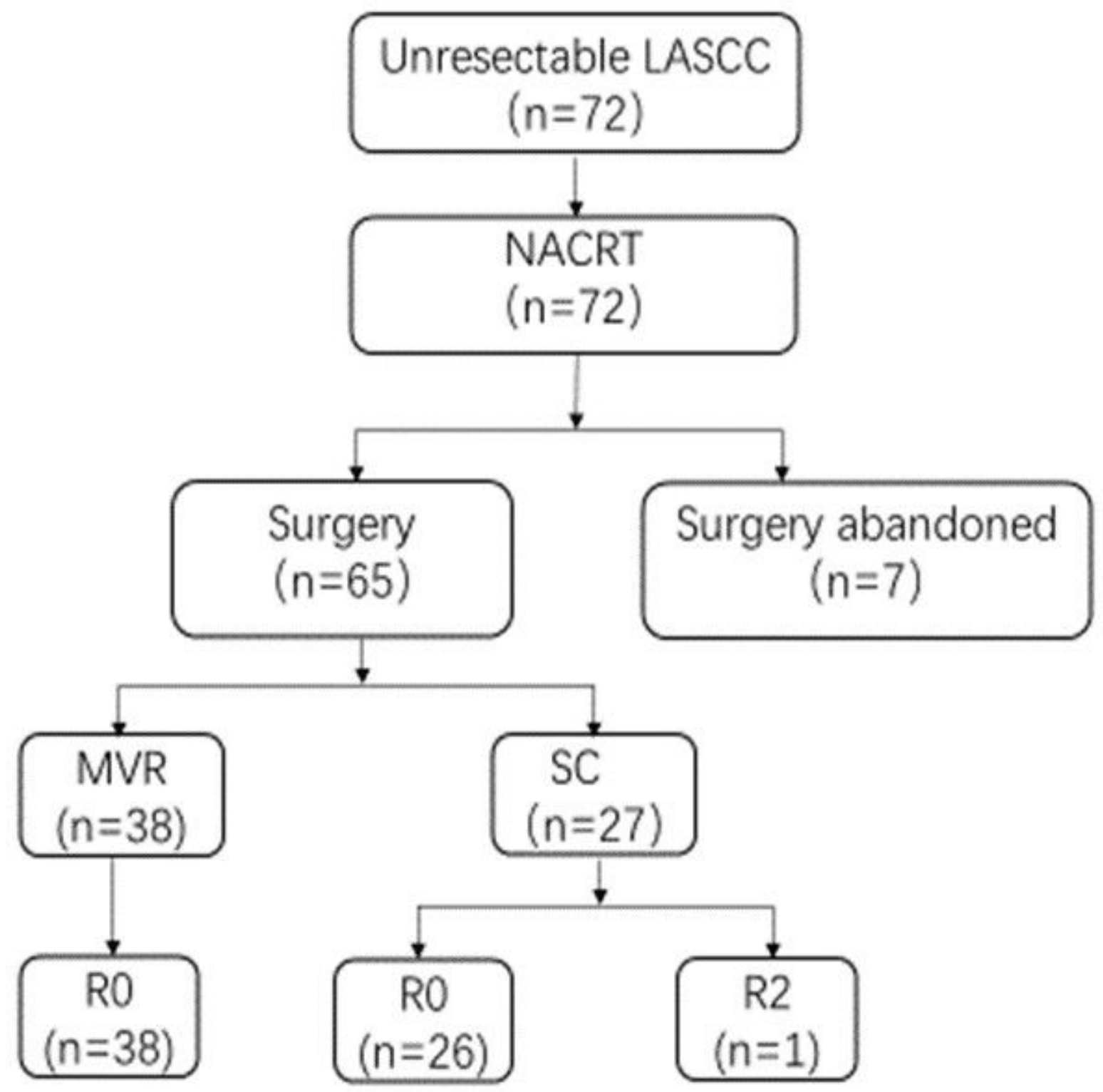

Figure 1

Treatment flow chart. 

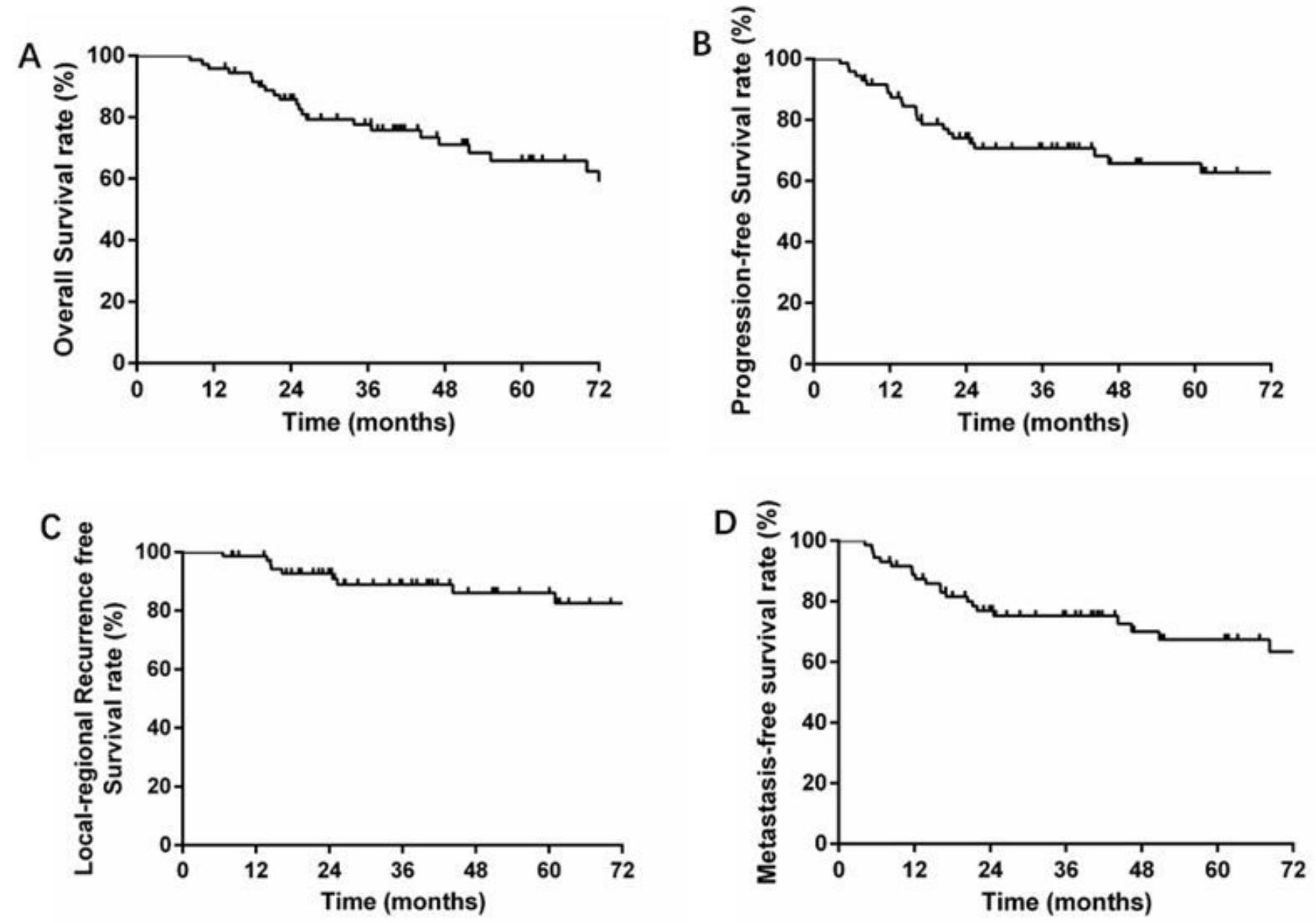

Figure 2

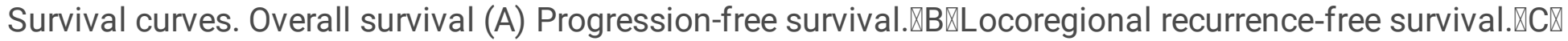
Metastasis-free survival.खD囚Survival curves of the 72 patients with unresectable LASCC. 
A
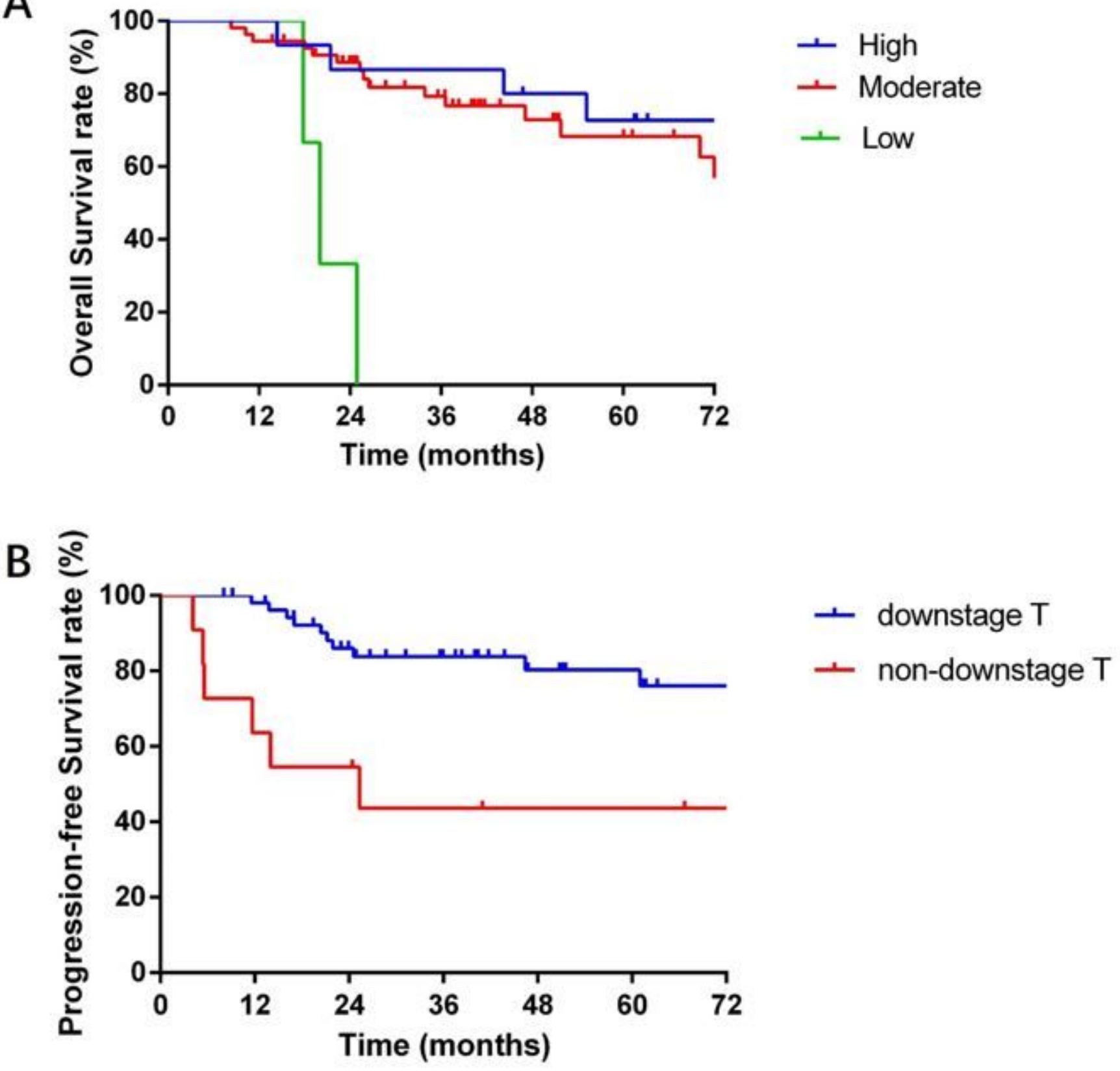

Figure 3

Subgroup analysis of survival. Overall survival by tumor differentiation $(A)(p=0.003)$ and progressionfree survival by downstaging $T(B)(p=0.027)$ in patients with unresectable LASCC with neoadjuvant chemoradiotherapy and surgery. 